\title{
Application of Image Processing Algorithms for Brain Tumor Analysis in 2D and 3D Leading to Tumor's Positioning in Skull: Overview
}

\author{
AYESHA AMIR SIDDIQI*, GHOUS BAKSH NAREJO**, SHEHNILA ZARDARI***, \\ MASHAL TARIQ****, AND SHEHLA ANDLEEB**** \\ RECEIVED ON 07.09.2016 ACCEPTED ON 22.11.2016 \\ ABSTRACT
}

\begin{abstract}
Segmentation of brain tumors has been found challenging throughout in the field of image processing. Different algorithms have been applied to the segmentation of solid or cystic tumors individually but little work has been done for solid cum cystic tumor. The papers reviewed in this article only deal with the case study of patients suffering from solid cum cystic brain tumor as this type of tumor is rarely found for the purpose of research. The research work conducted so far on this topic has been reviewed. The study begins with 2D (Two Dimensional) segmentation of tumor using MATLAB. It is then extended to study of slices of tumor and its volume calculation using open source software named 3D Slicer which represents the tumor in 3D. This software can intake the 2D slices and process them to give a combined 3D view. Various techniques are available in the software. According to the particular requirement an appropriate algorithm can be chosen. This paper gives a promising hierarchy for volume calculation of tumor and the three dimensional view. Further we can also find the position of tumor in the skull using the same software. This piece of work is a valuable guideline for the researchers interested in segmentation and three dimensional representations of different areas of human body. The models extracted out using the given algorithms can also be treated for matching and comparison of any future research. This will also aid surgeons and physicians in efficient analysis and reporting techniques.
\end{abstract}

Key Words: Segmentation, MATLAB, 3D Slicer, Brain tumor, Volume, Skull.

\section{INTRODUCTION}

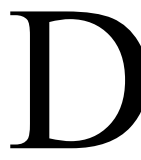
iseases like tumors are always found to be dangerous and are to be handled with special care. Tumor may be benign or malignant [1]. In any case if the tumor is found in the brain then expert physicians and surgeons have to make the proper treatment and its analysis. This is made by conducting the MRI (Magnetic Resonant Imaging) scan or CT (Computed Tomography) scans of the brain [2-3]. MRI

\footnotetext{
* Department of Telecommunication Engineering, Dawood University of Engineering \& Technology, Karachi.

** Department of Electronics Engineering, NED University of Engineering \& Technology, Karachi.

*** Department of Computer Science \& Software Engineering, NED University of Engineering \& Technology, Karachi.

**** Usman Institute of Technology, Karachi.
}

Mehran University Research Journal of Engineering \& Technology, Volume 36, No. 1, January, 2017 [p-ISSN: 0254-7821, e-ISSN: 2413-7219] 
is considered to be a very sensitive test and a single movement may cause the patient to go through the test again as the magnetic resonant imaging machine works on the principle of quantum mechanics dealing with spin of protons and neutrons [4]. There are different pulse sequences which are applied to samples resulting in the distinctive reconstruction of images. These are useful in generating different contrasts between the tissues, thus providing a wide variety of choices to the radiologists. Some commonly used MRI scans include T1weighted MRI, T2 weighted MRI and FLAIR (Fluid Attenuated Inversion Recovery) [5]. In T1 weighted image the image contrast and brightness are determined. On T1 weighted images fat appears bright and fluid dark. Whereas on $\mathrm{T} 2$ weighted images fluid appears bright and fat dark. Therefore, T1 weighted images are used to study solid part of tumor and T2 weighted images are used to study the cystic part. Other than these, FLAIR uses Inversion-Recovery Pulse Sequence. It is basically used to null signal from fluids. Signal from any particular tissue can be suppressed by selecting appropriate inversion time. It can be used in brain imaging to null the signals from CSF (Cerebral-Spinal Fluid). Work has been done to show the brain images into their respective Grey and White matters [6]. During MRI even a slight motion of the patient could produce artifacts on image [7]. It may be noise. The noise reduction filter is very important for the image enhancement [8]. Noise reduction then leads to segment out the ROI (Region of Interest). Segmentation turns out to be a decisive step in image processing [9]. It includes preprocessing techniques and different algorithms of images. There are two types of segmentation; one is under segmentation and the other is over segmentation. Under segmentation can be considered as tumor detection but with extra features (objects) which could result in false detection. In case of over segmentation the detection of tumor includes some other parts as well.
The doctors use to follow the traditional 2D technique for viewing the tumor part although 3D modernized algorithm may be applied without missed tumor detection. Automatic system for segmentation could be used. Whatever area is segmented out can be analyzed in 3D giving a complete view to the medical staff of the size and position of the tumor in the skull.

This review is carried out considering various research papers which give different techniques for $2 \mathrm{D}$ and $3 \mathrm{D}$ views and analysis. This paper will begin with review of 2D papers and followed by 3D analysis. Each paper with its results is studied individually.

\section{MATERIALS AND METHOD}

\subsection{Materials}

The material includes a dataset of cases of patients suffering from tumor which is solid and cystic both at the same time. The samples are taken from the laboratory, from GE 1.5 Tesla MRI with the DICOM (Digital Imaging and Communications in Medicine) software. Software used for 2D is MATLAB; whereas for 3D studies, 3D SLICER 3.6 is used. Contrast enhanced T1 and T2 weighted images are taken. 2D analysis is done from the axial view. All the work is carried out on Microsoft Windows XP (Professional), version 2002 with 2 GB of RAM. Intel Core 2 Duo, $1.83 \mathrm{GHz}$ processor.

\subsection{D Segmentation using T2 and T1 Weighted Images}

The first technique used for segmenting out tumor begins with morphological reconstruction of the images. The images are converted into JPEG (Joint Photographic Experts Group) with 100\% resolution from the original DICOM images in order to provide complete intensity pattern Fig. 1(a-b). They are grey scale images with $512 \times 512$ pixels. The gray scale of intensity is from 0 to 255 pixels. The axial view of MRI is taken as the tumor is 
visible maximum in this view whereas coronal and sagittal views can be taken as well [10]. Morphological reconstruction shows accurate results. Solid part is segmented using contrast enhanced $\mathrm{T} 1$ where as cystic part is taken from $\mathrm{T} 2$. The procedure begins by using a filter for noise reduction which is important for the image enhancement. Instead of taking the mean, the median of the neighborhood pixels are used to find the output using the default $3 \times 3$ neighborhoods as the mean filter resulted in merging the edges of tumor [10]. The texture of tumor is same as the rest of brain so the Mean filter results in blurring the image. To trace the boundaries automatically Sobel mask will be used which will give the information by using the method of convolution of two kernels [10]. Sobel results are more accurate than other filters. Other than the brain tumor, many edges were also detected which will be difficult to segment if any other filter was applied.

$$
h_{x}=\left(\begin{array}{lll}
1 & 0 & -1 \\
2 & 0 & -1 \\
1 & 0 & -1
\end{array}\right), h_{y}=\left(\begin{array}{ccc}
1 & 2 & 1 \\
0 & 0 & 0 \\
-1 & -2 & -1
\end{array}\right)
$$

The above kernels in Equation (1) are used to detect the horizontal $\left(h_{y}\right)$ and vertical $\left(h_{x}\right)$ edges.

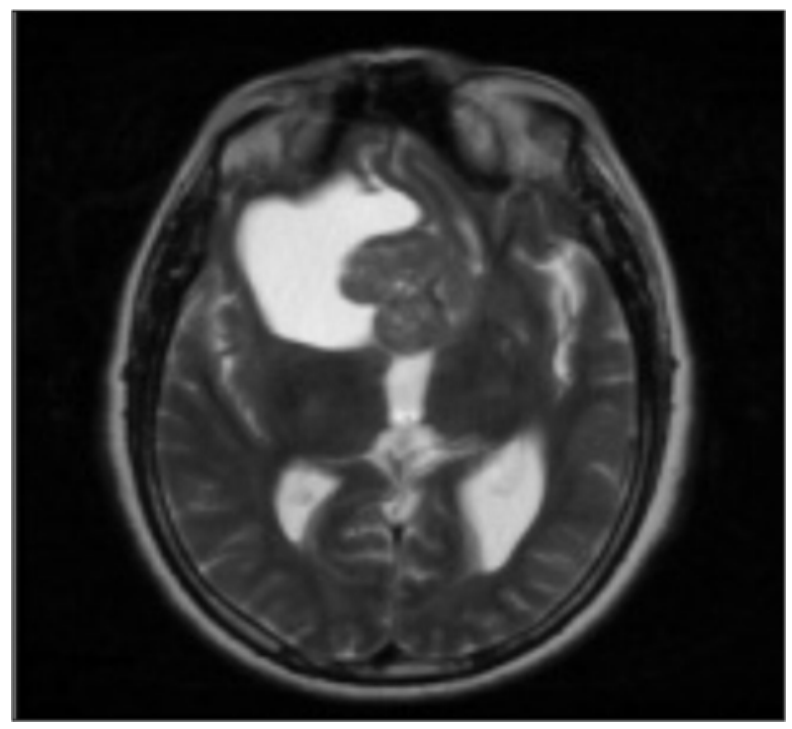

FIG. 1(a). T2 WEIGHTED
The kernels will be specified for horizontal and vertical edges respectively and then gradient method is applied to detect the edges [10]. Morphological reconstruction will be done initiating with thickening the boundaries by dilating using disk shape followed by eroding [12]. The morphological operations for Image Segmentation and Tracking Edges include procedures in which object undergoes the opening i.e. removing small objects and closing[13].The procedure of erosion involves removing small holes which is the operation essential for noise removal during morphological erosion and dilation. The command of imreconstruct (MATLAB) function uses the algorithm given in Vincent [14]. Opening using reconstruction gives better result. Further erosion restores the image. The challenging part is to make the solid and cystic part to appear at the same time and in the same way as shown in the MRI scan. For this purpose OR gate is used. It is its characteristic that if there is logic 1, results in output high. Fill the holes of the image using imfill (MATLAB) command which makes the tumor more prominent to become the ROI. The procedure concludes by the conversion of binary image to RGB (Red, Green and Blue) which undergoes the transparency making from where the detected tumor is superimposed on the MRI scan image. The whole procedure is illustrated by the

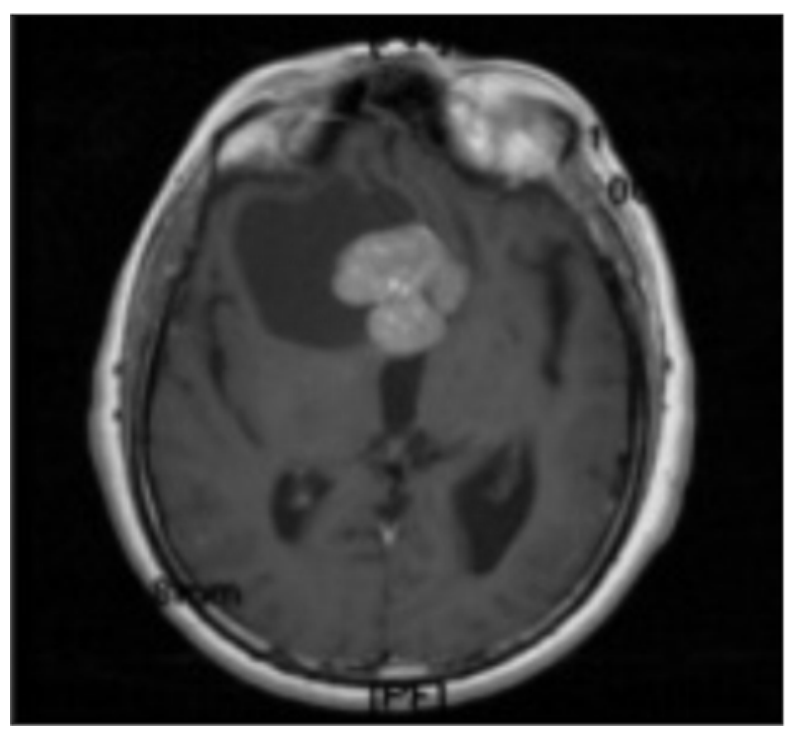

FIG.1(b). T1 WEIGHTED CONTRAST ENHANCED

Mehran University Research Journal of Engineering \& Technology, Volume 36, No. 1, January, 2017 [p-ISSN: 0254-7821, e-ISSN: 2413-7219] 
block diagram as shown in Fig. 2. The transparency which is superimposed on original image is shown in Fig. 3. The arrow is pointing at the tumor detected in Fig. 3.

\subsection{D Segmentation Using T1 Images}

The second technique is based on the study of intensities of the region of interest [15]. This method is applied on the T1 weighted images only. It begins with using the median filter which also has the advantage of edge keeping along with the noise removal. After filtering the edges are detected using the canny option of MATLAB [15]. Among the entire filter, this produces required and promising results. 0.005 And 0.15 are the two selected threshold

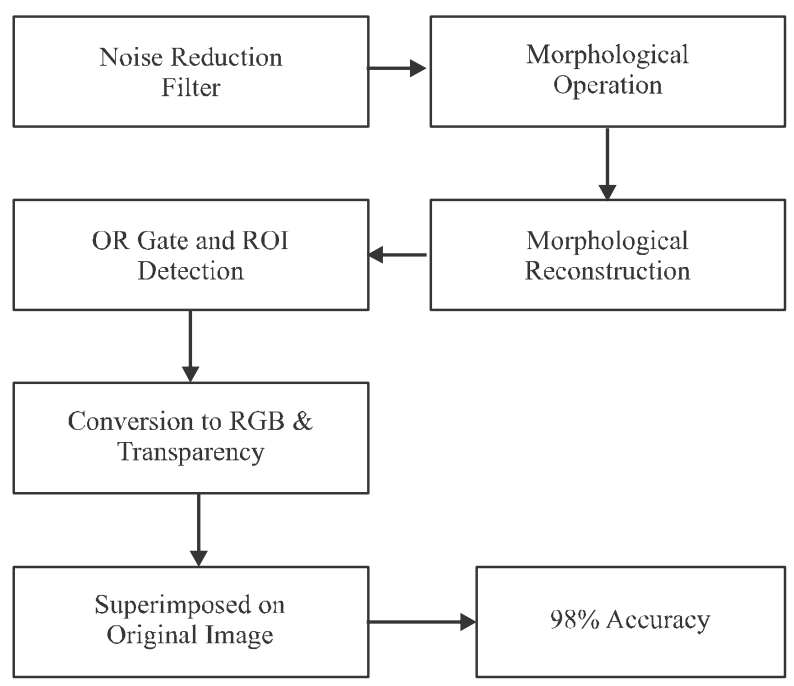

FIG. 2. BLOCK DIAGRAM FOR $1^{\text {ST }}$ TECHNIQUE values. These thresholds are applied on the basis of intensities.

In order to segment out the solid part convert the image to label from binary. An array of structure will be obtained. The main part of the technique lies in extracting the solidity of areas applying the command of region props (MATLAB). The commands applied on the basis of intensity and will result in labeled tumor.

The cystic part will be segmented by applying the region growing method [15]. Cystic part appears with other parts of brain. Apply the Bwareaopen (MATLAB) command on the image with cystic part to remove the unwanted region. Dilation and erosion technique will be used here.

Finally the OR logic operation, conversion RGB and to transparency in applied. The segmented tumor is then imposition in original one [15].In the block diagram red portion indicates the tumor detected.

In this technique it is to be kept noticed that as the tumor size changes the structure element will also change its size. Therefore, the threshold level will vary for different cases and has to be kept changing accordingly both for the solid and cystic part. The above methodology is illustrated as in block chart Fig. 4. Further a comparison of few similar algorithms with the above discussed algorithm is given in Table. 1 .

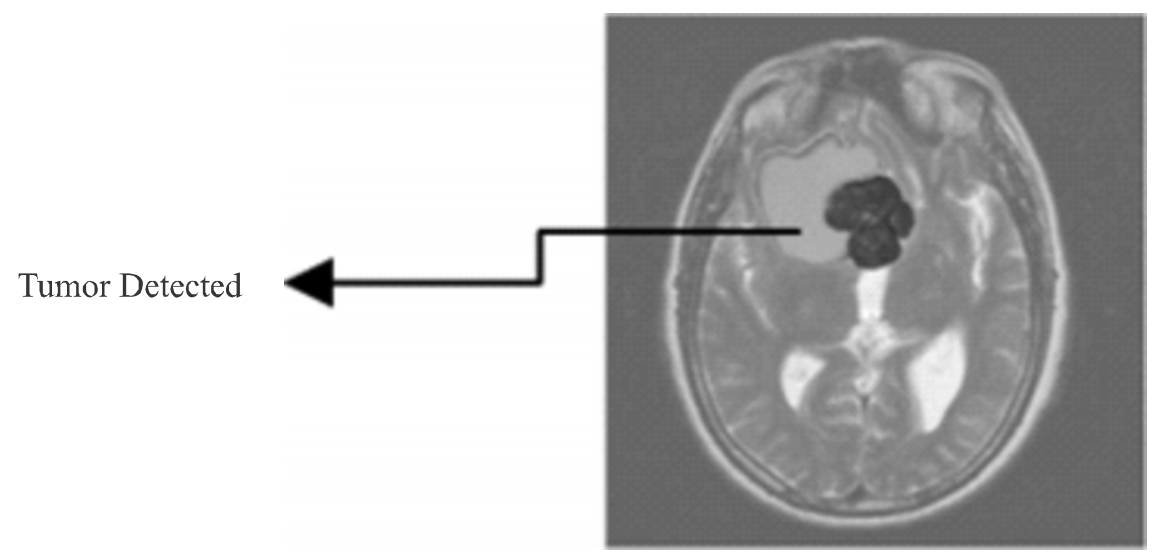

FIG. 3. FINAL RESULT 


\subsection{Technique for 3D Volumetric Representation of Tumor}

T2 weighted as well as contrast enhanced T1 weighted images are taken. The procedure initiates by loading all the volumes i.e. all the slices which are 19 in number (as taken from the MRI machine by the radiologist) into the 3D slicer [17] .In this scenario JPEG conversion is not required as the Slicer supports the DICOM images as they are. The images are then chosen independently for analysis from the Slicer libraries. The images are enhanced with respect to solid and cystic part appearing bright respectively on $\mathrm{T} 2$ and $\mathrm{T} 1$ contrast enhanced images by

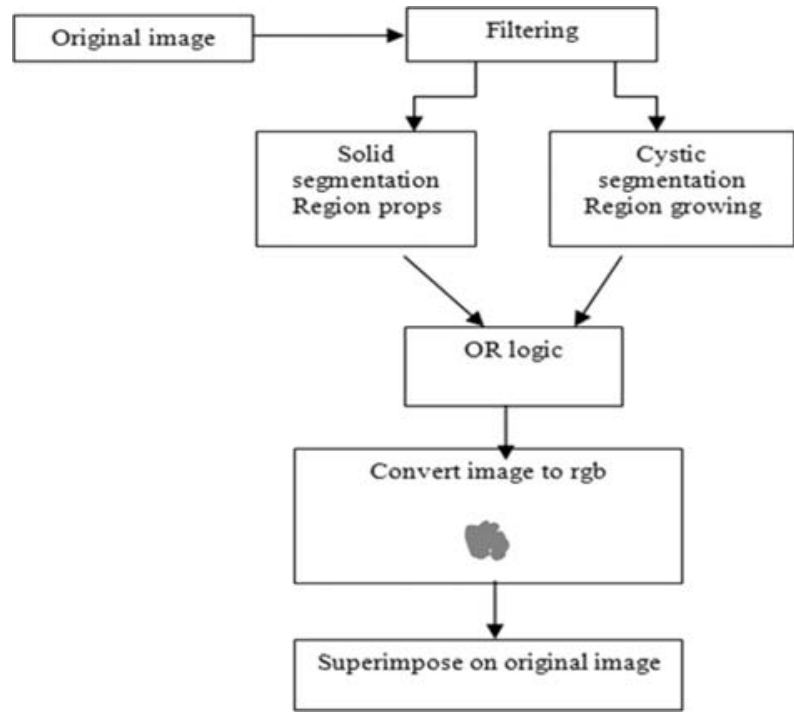

FIG. 4. 2D SEGMENTATION USING CONTRAST ENHANCED T1 IMAGES using the WLP (Window Level Preset) tool. After applying various techniques for segmenting out the desired part it has been found that label mapping method [17] gives promising results as compare to simple region growing method which selects a seed point and may result in over segmentation. This is considered to be the semi automatic part in which the boundary of the solid or cystic part is traced manually and then the traced area is filled by applying a map of color to it. The holes are filled automatically by joining with the neighborhood cells in the labeled region. This procedure is followed for all the slices in which the tumor is visible. The labeled parts are then further loaded for model making. This provides a $3 \mathrm{D}$ view of both the solid and cystic part. The model maker initially converts each label map to binary and then it nullifies all the part except the labeled part. This portion then undergoes marching cubes model [18] for volume acquisition. The 3D models for solid and cystic parts are then stored in the models module which makes a hierarchy of the results for one particular case and it displays the tumor as a whole when called. The volume of the tumor is displayed in this module which stores the complete information about the case. Volume of the cyst turns out to be $69514 \mathrm{~mm}^{3}$ and the volume of solid is $25286 \mathrm{~mm}^{3}$ respectively shown in Fig. 5.Total processing time is 5 seconds after segmentation. The steps of this methodology can be viewed in the Fig. 5(a-d).

TABLE 1. COMPARISON OF 2D SEGMENTATION ALGORITHMS

\begin{tabular}{|c|c|c|c|}
\hline Author(s) & Method & Accuracy (\%) & Time (Seconds) \\
\hline Corso, et. al.[6] & Morphological Watershed Segmentation & 70 & - \\
\hline Mashal, et. al.[10] & Morphological Reconstruction Based Segmentation & 98.95 & 19.25 \\
\hline Mashal, et. al.[15] & Threshold and Region Growing Based Segmentation & 98 & 10 \\
\hline Macenko, et. al.[16] & Integrated Bayesian Model Classification & 90 & - \\
\hline
\end{tabular}

Mehran University Research Journal of Engineering \& Technology, Volume 36, No. 1, January, 2017 [p-ISSN: 0254-7821, e-ISSN: 2413-7219] 


\subsection{Technique for 3D Volumetric Representation of Human Skull with Tumor}

This study is further extended to the modeling of skull from original data set. $\mathrm{T} 1$ or $\mathrm{T} 2$ any of the images can be taken as in both of them the boundary or the edges of the skull in all the slices is visible. It will give the presence of tumor in the skull and analyze what trajectory is to be followed from external of the brain to the tumor. This is started by loading all the images into slicer libraries [19]. The images are then directly loaded in to the editor module for mapping of complete areas visible along with the edges. The surface model maker will then combine all the labeled slices to give the complete view of the skull [20]. Here triangle smoothing can be applied to have a neat view of the skull. This model is also stored with its tumor in the model hierarchy tree present in the models module. Call all the parts one by one and will get a complete view of the original skull bearing tumor which is shown in Fig. 6. The algorithm turns out to be simpler
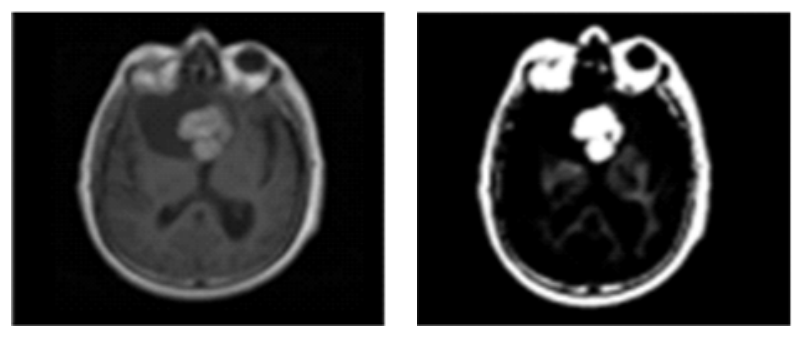

(a) Image Enhancement
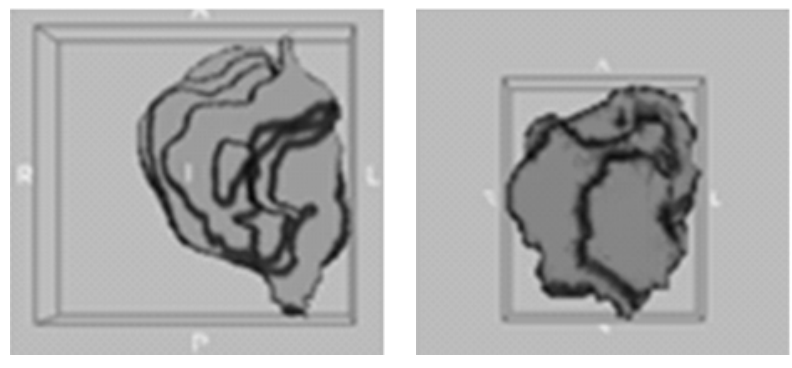

(c) Surface Model Making as compared to neurofuzzy techniques applied for the segmentation of tumor and then its modeling in order to show the lesion in the brain [21]. The objective of neurosurgical resection of brain lesions is, maximal cutting out of tumor or lesion with minimal permanent damage to the neighboring normal brain tissue [22]. It can be made possible once the tumor distance and location from the skull is visible.

\section{CONCLUSIONS}

All the above given algorithms have been applied to various cases and gives good results with $98 \%$ efficiency on an average.

The segmentation being an important part of image analysis is done effectively in 2D . The study of $3 \mathrm{D}$ analysis also gives promising results of volume calculation and the model of tumor. The tumor representation in skull will be helpful for the surgical purposes. The skull modeling will facilitate the surgeons in biopsy and radiotherapy.
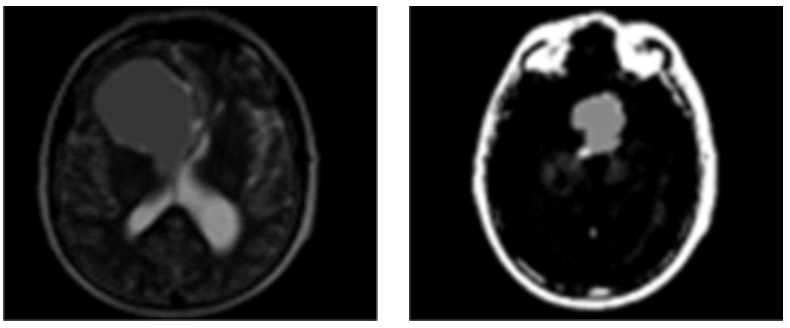

(b) Label Map Segmentation

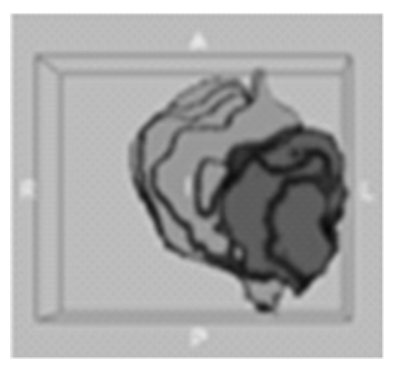

(d) Result

FIG. 5. STEPWISE 3D MODELLING OF SOLID AND CYSTIC TUMOR

Mehran University Research Journal of Engineering \& Technology, Volume 36, No. 1, January, 2017 [p-ISSN: 0254-7821, e-ISSN: 2413-7219] 


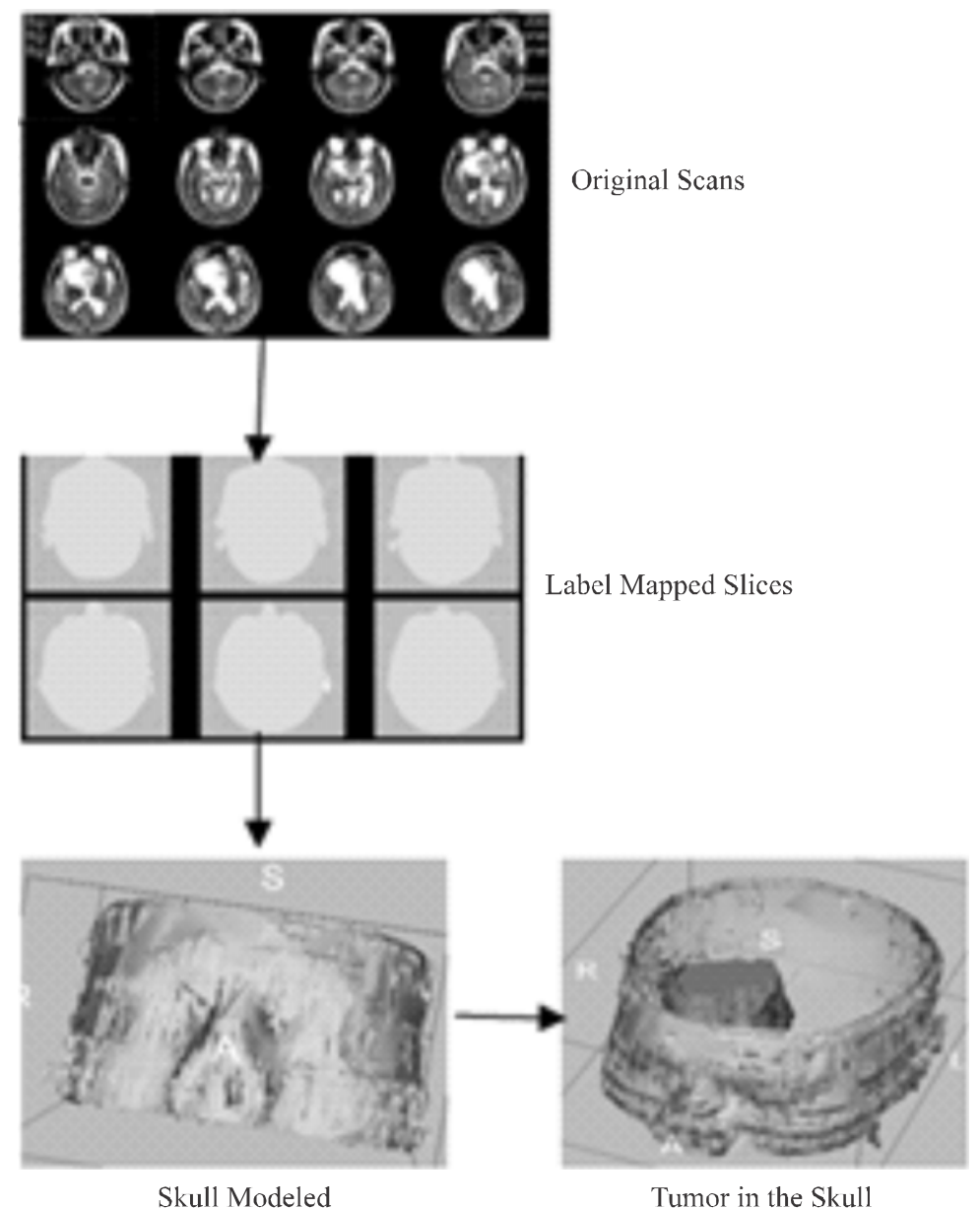

FIG. 6. SKULL 3D VIEW

\section{FUTURE WORK}

Research work can be done to design a complete automatic system. This review of papers will be helpful for the researchers for comparing their results and gives the significance of 3D Slicer software used in neurosurgical experiments, which is extremely good for counter checking the algorithms. It will also help in the designing of a tool approaching the tumor to take biopsy material as it gives the trajectory from skull to tumor.

\section{ACKNOWLEDEMENTS}

Authors thank Dr. Saad Qazi, NED University of Engineering $\&$ Technology, Dr. Munnawar Hussain, and Dr. Raffique Khanani, Dow University of Health \& Sciences, and Dawood University of Engineering \& Technology, Karachi, Pakistan, for providing immense support, guidance and help.

\section{REFERENCES}

[1] Chris, A.C., Alex, P.Z., and Alan, C.E., "Automatic Generation of Training Data for Brain Tissue Classification from MRI", Medical Image Computing and Computer-Assisted Intervention-book, McGill University, Canada, 2002

[2] Moon, N., Bullitt, E., Leemput, K., and Gerig, G., "Automatic Brain and Tumor Segmentation", MICCAI, Medical Image Computing and Computer-Assisted Intervention-book, LNCS Volume 2489, pp. 372-379, Canada, 2002.

[3] Somasundaram, K., and Kalavathi, P., "Analysis of Imaging Artifacts in MR Brain Images", Oriental Journal of Computer Science \& Technology, [ISSN: 0974-6471], Volume 5, No. 1, pp. 135-141, India, June,2012. 
[4] Keller, P., "Basic Principles of MR Imaging", Book on GE Medical Systems, Milwaukee, 1988.

[5] Eric, C.W., Thomas, T.L., Wen-Ming, L. Lawrence, R.F., and Richard, B.B., "T1 and T2 Selective, Method for Improved SNR in CSF-Attenuated Imaging: T2-FLAIR", Journal on Magnetic Resonance in Medicine, Volume 45, pp. 529-532, California, 2001.

[6] Corso, J.J., Sharon, E., Dube, S., El-Saden, Z., Sinha, U., and Yuille, A., "Efficient Multilevel Brain Tumor Segmentation with Integrated Bayesian Model Classification Inverse Filtering", IEEE Transactions on Medical Images, Volume 27, pp. 629-640,USA, 2008.

David, T.G., Arya, N., Ron, K.,Noby, H., Lauren, J., O’Donnell, W., Eric, L., Grimson, F.A., Jolesz, P.M., Black, W.M., and Wells-III, "An Integrated Visualization System for Surgical Planning and Guidance Using Image Fusion and an Open MR", Journal of Magnetic Resonance Imaging, Volume13, pp. 967-975, 2001.

George, E.B., "MRI Brain Image Enhancement Using Filtering Techniques", International Journal of Computer Science \& Engineering Technology, [ISSN: 2229-3345], Volume 3, No. 9, September, 2012.

Kaleem, K., Sanaullah, M., Hussain, M.A., Jaffar, M.A., Tae-Sun, C., "Segmentation of Brain Tumor Tissue Using Marker Controlled Watershed Transform Method", Emerging Trends and Applications in Information Communication Technologies, Journal of Communications in Computer \& Information Science, Volume 281, pp 222-227,Berlin, 2012.

[10] Tariq, M., Khawaja, A., and Hussain, M., "Image Processing with the Specific Focus on Early Tumor Detection", International Journal of Machine Learning and Computing, Volume 3, No. 5, Singapore, October, 2013 .

[11] Lau, P.Y., Voon, F.C.T., and Ozawa, S., "The Detection and Visualization of Brain Tumors on T2-Weighted MRI Images using Multi Parameter Feature Blocks", Proceedings of IEEE $27^{\text {th }}$ Annual Conference on Engineering in Medicine and Biology, pp. 5104-5107, Shanghai, 2005.

[12] Chowdhry, S., Babu, G.A., and Rani, M.U., "Gray-Level Morphological Operations for Image Segmentation and Tracking Edges on Medical Applications", International Journal of Computer Science and Network Solutions [Online], Volume 9, pp. 131-136, July, 2009.
[13] Jones, R., and Svalbe, I., "Algorithms for the Decomposition of Gray-Scale Morphological Operation", IEEE Transactions on Pattern Analysis Machine Intelligence, Volume16, pp. 581-588, Illinios,USA, 2004.

[14] Vincent, L., "Morphological Grayscale Reconstruction in Image Analysis: Applications and Efficient Algorithms", IEEE Transactions on Image Processing, Volume 2, pp. 176-201, USA, 1993.

[15] Tariq, M., Andleeb, S., and Siddiqi, A.A., "Effective Procedure of Solid cum Cystic Brain Tumor Segmentation through MATLAB, the Environment Monitor", Journal of Engineering, Sciences \& Technology, Volume XV, [ISSN1684-8012], Pakistan, May-June 2015.

[16] Macenko, M., Celenk, M., and Ma, L.M., "Lesion Detection using Morphological Watershed Segmentation and Model Based Inverse Filtering", International Conference on Pattern Recognition, Volume 4, pp. 679682, Hong Kong, 2006.

[17] Siddiqi, A.A., Khawaja, A., and Tariq, T., "3DVolume Representation of Brain Tumor Using Image Processing”, IEEE Conference on Signal and Image Processing Applications, Kaula Lumpur, Malaysia, 16-18 November, 2011.

[18] Timothy, S.N., and Yi, H., "A Survey of the Marching Cubes Algorithm", Journal on Computers \& Graphics, Volume 30, pp. 854-879, North Carolina, 2006.

[19] Siddiqi, A.A., Khawaja, A., and Tariq, M., “3D Representation of Human Skull Bearing the Tumor Using Image Processing”, IEEE International Conference on Computational Intelligence, Communication Systems and Networks, Thailand, 24-26 July,2012.

[20] Murugavalli, S., and Rajamani, V., “An Improved Implementation of Brain Tumor Detection Using Segmentation Based on Neuro Fuzzy Technique", Journal of Computer Science, Science Publication, Volume 3, No. 3, pp.841-846, 2007.

[21] 3D Slicer Documentation, User Guide, www.slicer.org.

[22] Tharin, S.M.D., "Functional Brain Mapping and its Applications to Neuro Surgery", Department of Neurosurgery, Brigham and Women's Hospital, Harvard Medical School, Boston, Massachusetts, August 30, 2006 